\title{
The unexpected effects of added ligands on the addition of phenyllithium to $E$-cinnamaldehyde in THF
}

\author{
Alvaro J. Vázquez and Norma Sbarbati Nudelman* \\ Depto. Química Orgánica, Facultad de Ciencias Exactas y Naturales, Universidad de Buenos \\ Aires, Pab. II, P. 3, Ciudad Universitaria, 1428 Buenos Aires, Argentine \\ E-mail: Nudelman@qo.fcen.uba.ar
}

Dedicated to Professor Rosa Muchnik de Lederkremer

\begin{abstract}
The effect of donor ligands, usually expected to enhance the reactivity of organolithiums, was studied on the addition of PhLi to E-cinnamaldehyde in THF, under conditions that lead the reaction toward the production of 1,3-diphenylpropanone. It was observed that in the presence of TMEDA and HMPT, the rate of that reaction becomes slower than in the absence of ligands; the effect of HMPA was even more spectacular, at concentration [HMPA]: [PhLi] $\geq 4$ the reaction becomes almost completely inhibited. These results show the complexity of solvation effects on the reactions of organolithiums and how substrate-reagent-ligand-solvent interactions are usually specific for the system under study.
\end{abstract}

Keywords: Phenyllithium, aggregation states, addition to $\alpha, \beta$-unsaturated compounds, tandem reactions, ligands, additives

\section{Introduction}

The seminal work by Seebach in 1984, followed by a second work in 1988, alerted synthetic chemists on the effects of aggregation of organolithium compounds.1 This feature is currently being used as an excellent tool to modify the reactivity, and also the regio- and stereochemical course of reactions, as shown by abundant recent literature.2 Aggregation states can be usually tuned up by a careful choice of solvent, co-solvent and/or by the addition of different types of ligands.3 Lithium amides are also known to be aggregated, $6 \mathrm{Li}$ and ${ }_{13} \mathrm{C} \mathrm{NMR}$ spectroscopic studies as well as kinetic determinations, have shown a wide spectrum of aggregated structures, included mixed aggregates.4,5,6 We have recently reported how mixed aggregates with the amine acting as a proton donor can be constructively used for the synthesis of complex molecules.7 Collum and Cow. have recently reported the complex relationship of solvent binding constants 
and relative reactivities toward 1,2-additions.8

In recent years, we have been involved in studying the mechanisms of addition of organolithium compounds to $\alpha, \beta$-unsaturated compounds; by careful choice of different variables, it was possible to lead the reaction toward the production of unexpected intermediates, able to undergo further tandem sequences.9 Tandem reactions are currently being considered suitable alternatives for sustainable organic synthesis. Since several bonds could be formed in one sequence without isolating the intermediates or changing the reaction conditions, 10 the amounts of solvents, reagents, adsorbents and energy are dramatically decreased, compared with stepwise reactions.11 Because of their synthetic relevance, tandem reactions involving organolithium compounds have been recently reviewed.12 The fine sensitivity of the reaction of $\alpha, \beta$-unsaturated compounds with $\mathrm{PhLi}$ to the reaction conditions, made us suspect that aggregation structures could be involved. The purpose of the present work was to examine the effect of additives able to change the aggregation states of the reagent.

\section{Results and Discussion}

The reaction of E-cinnamaldehyde, 1, with phenyllithium in THF was studied under several reaction conditions. It was found that THF is the more suitable solvent to afford the addition product, and the less sensitive to the temperature variations.13 Thus, when PhLi: $[1]=1$, and $\mathrm{T}=$ $-78^{\circ} \mathrm{C}$ the expected addition product 1,3-diphenyl-3-propen-1-ol, 2, is formed in few minutes in high yields. On the contrary, for $\mathrm{PhLi}:[1]=2$, at room temperature and long times of reaction, a more interesting compound, the 1,3-diphenylpropanone, $\mathbf{3}$, is the main reaction product; traces of the chalcone 4 , are also formed in variable amounts.9 (equation 1).

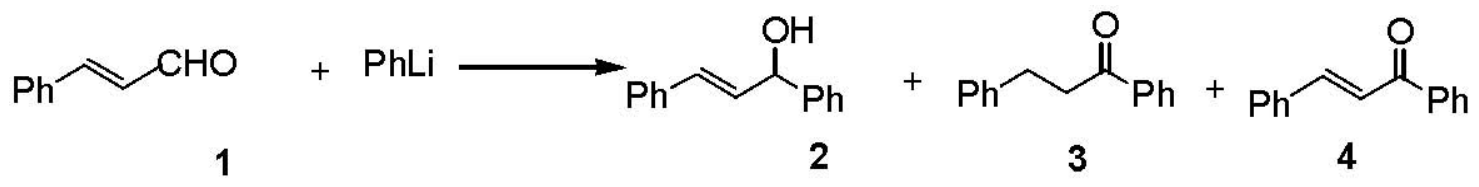

The sensitivity of the reaction to concentration and solvent effects, could indicate that the subtle equilibrium between monomer and oligomers of $\mathrm{PhLi}$ could be responsible for the observed results. There is abundant evidence in the literature that $\mathrm{PhLi}$ forms a mixture of dimer and monomer in THF; this has been confirmed by recent determinations.14 The reported value for the equilibrium constant is $\mathrm{Keq}_{\mathrm{eq}}=$ [dimer] / [monomer $]_{2}=40 \mathrm{M}-1$, at $-75 \mathrm{oC} .15$ On the other hand, it has been demonstrated, especially by NMR spectroscopy, that the addition of tetramethylethylendiamine (TMEDA) and related diamines, strongly influences organolithium structures.16 The capacity of TMEDA to reduce organolithium aggregation and elicit high reactivities is a common understanding among synthetic organic chemists, although this 
assumption has been recently questioned.17 Collum and coworkers' investigations in the last years made important contributions on the way how polyfunctional solvents influence some organolithiums; their studies showed that in the 1,2-addition of $\mathrm{PhLi} / \mathrm{TMEDA}$ to a functionalized imine monomer-based transition structures are involved; 8 while in the addition of BuLi/TMEDA the rate studies revealed a complex scenario with four independent mechanisms in which both monomer- and dimer-based pathways are involved.18 A cooperative solvation TMEDA/Et $2 \mathrm{O}$ was observed in this last case. 18

Table 1. Reaction of E-cinnamaldehyde, 1, with PhLi in THF. Effects of added ligandsa

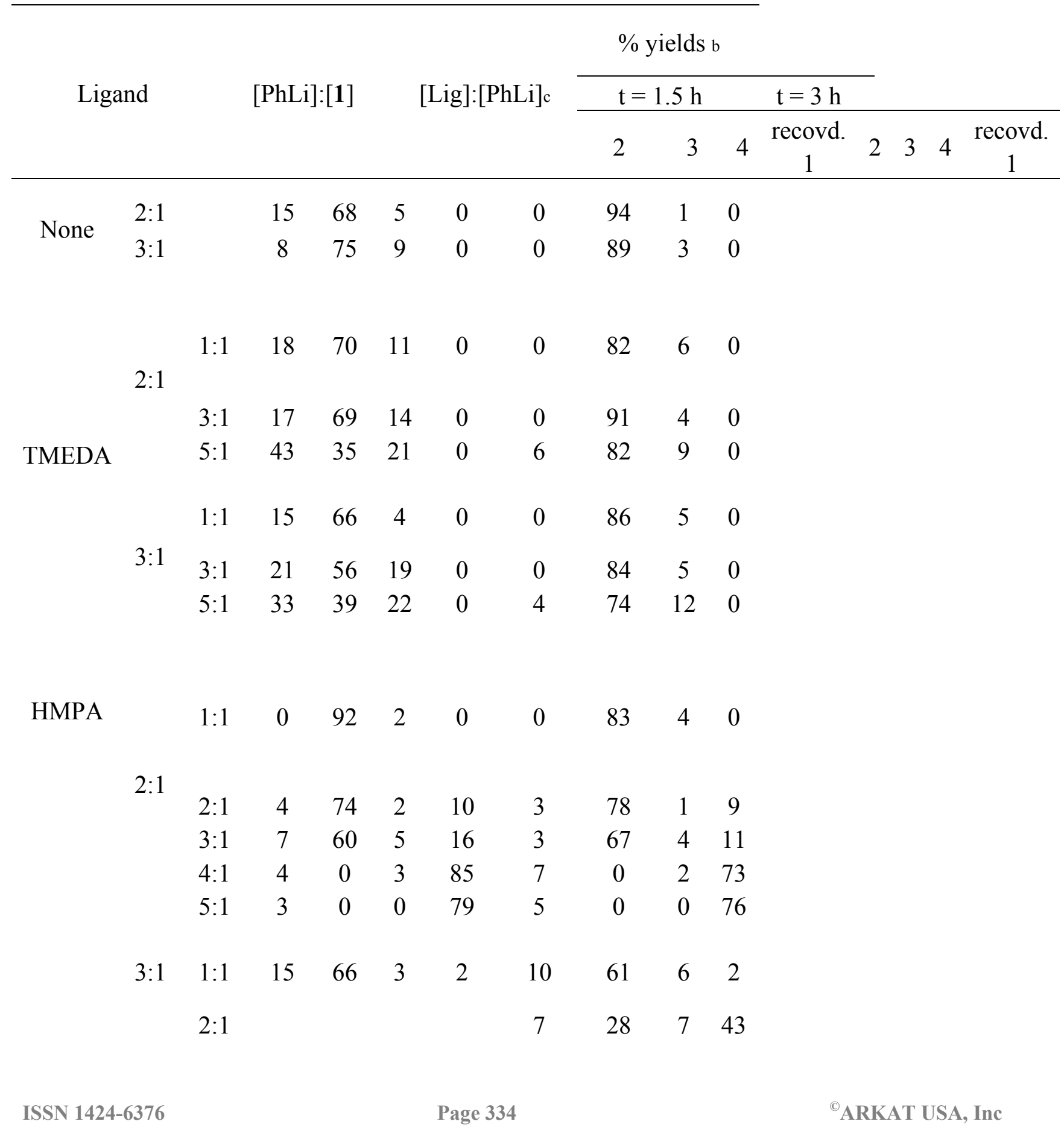




\begin{tabular}{ccccccccccc} 
& & $3: 1$ & 23 & 0 & 8 & 54 & 16 & 0 & 5 & 59 \\
\multirow{3}{*}{ HMPT } & $2: 1$ & $0.5: 1$ & 14 & 67 & 16 & 0 & 0 & 90 & 5 & 0 \\
& $2: 1$ & 19 & 50 & 19 & 0 & 0 & 88 & 3 & 0 \\
& $3: 1$ & $1: 1$ & 2 & 81 & 10 & 1 & 0 & 80 & 10 & 0 \\
\hline
\end{tabular}

$\mathrm{a}[\mathbf{1}]=0.07 \mathrm{M}$. Reactions carried out at room temperature. $\mathrm{b} \mathrm{t}=$ reaction time. $\mathrm{c}$ lig $=$ ligand

Based on the above grounds, TMEDA was the first complexing ligand to be examined in the reaction of $\mathrm{PhLi}$ with $\mathbf{1}$, under the conditions that lead the reaction toward the preferred production of compound $\mathbf{3}$. Results in Table 1 show that in the absence of additives the reaction is complete, $\mathbf{3}$ is the main reaction product, and no $\mathbf{1}$ is recovered. Since this work was carried out to elicit mechanistic insights, GC calibration curves were determined to assure accurate product concentrations using durene as subrogate; each run was carried out at least by duplicate. If the reaction is quenched after $1.5 \mathrm{~h}, \mathbf{3}$ is the main product, and $\mathbf{2}$ and $\mathbf{4}$ are formed in variable amounts. The yields of $\mathbf{3}$ increase at $3 \mathrm{~h}$ reaction time, $\mathbf{2}$ was not found and $\mathbf{4}$ appeared in low amounts, unreacted 1 was not observed. These results were interpreted as that the rate of formation of $\mathbf{3}$ is slower, and that it is mainly formed through the intermediates producing $\mathbf{2}$ at short reaction times.

In the presence of TMEDA, the yield of $\mathbf{3}$ diminishes in all cases, though the reaction is also complete in the sense that no $\mathbf{1}$ is recovered. For reaction times of $1.5 \mathrm{~h}$, it can be observed that when the ratio $[\mathrm{PhLi}]:[\mathbf{1}]=2$, the production of the conventional addition product, $\mathbf{2}$, increases as long as the proportion [TMEDA]:[PhLi] increases, and it becomes the main product for $[$ TMEDA $]:[\mathrm{PhLi}]=5$. Similar results were also observed when the ratio $[\mathrm{PhLi}]:[1]=3$. On the basis of the common understanding that solvation by TMEDA promotes deaggregation of the dimer, these results could indicate that the 1,2-addition product $\mathbf{2}$, is mainly formed through monomer $\mathrm{PhLi}$, while formation of $\mathbf{3}$ would require $(\mathrm{PhLi}) 2$. 


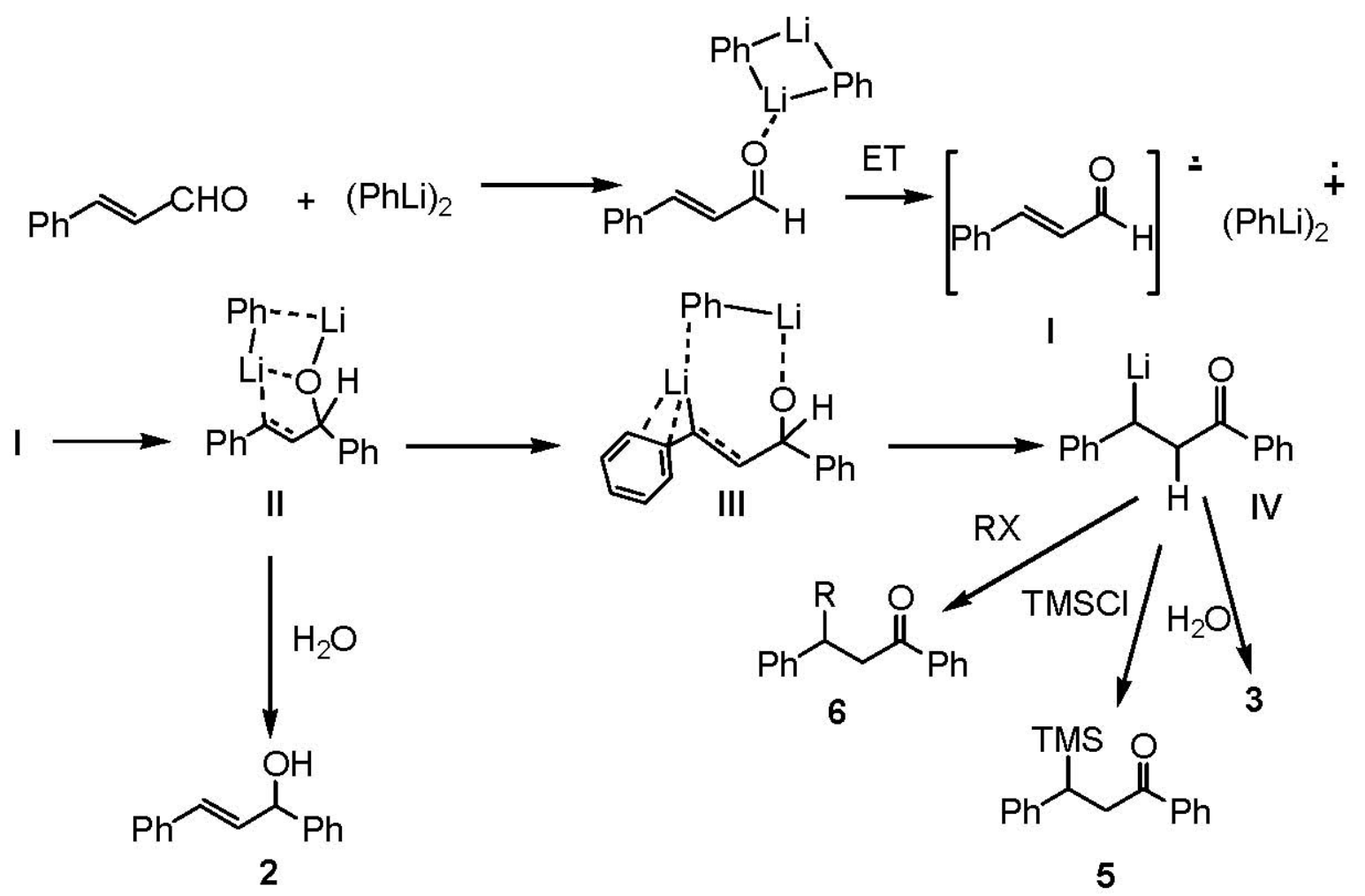

\section{Scheme 1}

A mechanism of reaction consistent with this interpretation is shown in Scheme 1. Although different species are certainly present in the reaction mixture, for the sake of simplicity, only the unsolvated dimer is considered. The initiation step is complexation of PhLi to $\mathbf{1}$, by a precoordination of the lithium atoms to the carbonyl oxygen. Complex formation between the reactants has been also invoked in recent examinations of how lithiations actually occur,19 specially when the organic precursor contains a functionality such as a $\mathrm{C}=\mathrm{O}$ or $\mathrm{P}=\mathrm{O}$ unit.20,21 The next step would be an electron transfer from phenyllithium to $\mathbf{1}$, giving the radical anion-radical cation pair I. Electron transfer from phenylithium to carbonyl compounds22,23 and to other electron aceptor functionalities 24 have been recently proved to be the first step in addition reactions. The unique feature of the present reaction is the coordination of the second lithium atom to the $\beta$-carbon giving a seven-membered cyclic intermediate II followed by a slow proton transfer from the electron deficient carbonyl carbon to the oxygen producing the more stable intermediate III. An additional effect of TMEDA in the present reaction would be slow down the rate of conversion to III which is the r.d.s. for the formation of $\mathbf{3}$. We have calculated the transition state from the $\mathbf{I I} \rightarrow$ III conversion by semi-empirical methods and found to be a real transitition state with the $\mathrm{H}$ atom vibrating between both structures.25 Detachment of a PhLi moiety forms IV, the lithiated precursor of 3 . Working up the reaction with $\mathrm{D}_{2} \mathrm{O}$ gives $100 \% \beta$ deuterated 3, which proves the presence of $\mathbf{I V}$ in the reaction mixture. Similarly, treatment of IV, with TMSCl afforded the TMS derivative, 5. The usefulness of the $\beta$-carbon lithiated precursor 
for the preparation of $\beta$-alkyl-substituted-dihydro-chalcones, $\mathbf{6}$, by tandem reaction has been previously reported.9,12

The effects of addition of hexamethylphosporamide (HMPA) were then studied. HMPA is a highly polar, aprotic solvent and its usefulness stems from its ability to coordinate to lithium very strongly, a recent measurement reports that it coordinates approximately 300 times more strongly than THF.26 HMPA enhances the reactivity of carbanions, amides, and enolates and can help effect difficult metalations.27 Strong complexation to lithium weakens the C-Li interactions and increases the fraction of SIP (separated ion pairs) present, with a great enhancement of nucleophilic reactivity.28 Effects of HMPA altering the regiochemistry of organollithium additions26b and metallations14a have been also reported. The results using HMPA in the present study are shown in Table 1 . With small amounts of the polar additive, i.e. at $[\mathrm{PhLi}]:[\mathbf{1}]=2$, and [HMPA]:[PhLi] $=1$, the rate of formation of $\mathbf{3}$ seems to be increased, since no $\mathbf{2}$ was found even at $\mathrm{t}=1.5 \mathrm{~h}$. On the contrary, when [HMPA]:[PhLi] $=2$, or 3, the decrease in the yield of 3 was significant, but it was not balanced by a similar increase in the yield of $\mathbf{2}$, as observed when TMEDA was used, and 10\% and 16\%, respectively, of unreacted 1 weres recovered after $3 \mathrm{~h}$. The effect of adding HMPA in a proportion [HMPA]:[PhLi] $=4$, is more spectacular, no 3 was formed under these conditions; the run was repeated several times to assure the data. Only small amounts of $\mathbf{2}$ and $\mathbf{4}$ were formed, a big amount of unreacted $\mathbf{1}$ was recovered. When HMPA was used in 5 times the $\mathrm{PhLi}$ concentration, similar results were observed: no $\mathbf{3}$, and only slight amounts of 2 were produced, the run was repeated by triplicate. When $\mathrm{PhLi}$ is more in excess, $[\mathrm{PhLi}]:[\mathbf{1}]=3$, and [HMPA]:[PhLi] $=1$, more alcohol is produced at expenses of $\mathbf{3}$. The amount of 3 diminishes as [HMPA] increases, becomes nul when [HMPA]:[PhLi] = 3, 2 is the main product. (Though the poor conversion of 1 under relatively high [HMPA] could, in principle, make one suspect as derived from adventitious water, the spectacular change from 3:1 to 4:1 (verified by triplicate), clearly indicates a specific HMPA effect).



7

These results could be indicative that, like in the case of TMEDA, the monomer reagent is likely involved in the 1,2-addition product probably through monomer transition structures. On the contrary, as shown in the Scheme, the reaction mechanism for the formation of $\mathbf{3}$ would require "free" dimer PhLi (likely solvated by THF). The spectacular effect of large proportions of HMPA can be interpreted as due to competitive progressive detachment of THF molecules from the Li atom, being replaced by HMPA moieties. Different HMPA solvated species would 
be formed, up to the tetrasolvated dimer shown by 7 . The steric hindrance at the reaction center would inhibits formation of intermediates II-IV, only relatively small amounts of intermediate I would be formed likely by the monomer PhLi in equilibrium with the dimer. This explanation is also consistent with the increase in the yield of 2 . Stoichiometric complexation of aryllithiums by HMPA15 and effects of steric hindrance in organolithium-mediated ketone and imine deprotonations have been previously reported.29

A few runs were then carried out in the presence of HMPT (hexamethyl phosphorous triamide). The results gathered in Table 1, show effects that are similar to those determined using TMEDA as ligand and can be interpreted in the same way.

The mechanism shown in Scheme 1 is a simplified picture of the complex species that are likely involved. Though the present results alone cannot be considered compelling evidence of the existence of the tetrasolvated dimer, they are useful to show that the effects of donor ligands are difficult to predict and in some cases they could diminish the reactivity of organolithiums and even inhibit the reaction. The observed effects on the addition of $\mathrm{PhLi}$ with $\mathbf{1}$, under the conditions producing the tandem addition-lithiation-alkylation sequence, are the consequences of complex substrate-reagent-solvent-ligand interactions, which are specific to the studied reaction.

\section{Experimental Section}

General Procedures. All reactions involving organolithium reagents were carried out by using standard techniques for the manipulation of air and water-sensitive compounds.30 All glassware, syringes and needles were dried in a oven and cooled in a dessicator. All compounds reported here were fully characterized by melting point (when applicable), mass spectrometry (using a gas chromatograph coupled to the BG-Trio-2-mass spectrometer), and nuclear magnetic resonance spectroscopy (determined on a Bruker 200 spectrometer and a Bruker 500 spectrometer) as described before.9 The GC analyses were carried out on a 5890 Hewlett-Packard gas chromatograph, equipped with FID detector and with HP-5 column.

Materials. Hexane and THF (HPLC grade) were distilled from dark blue solutions of sodium/benzophenone ketyls, under nitrogen, immediately prior to use. Commercial $E$ cinnamaldehyde was distilled at reduced pressure prior to use. TMEDA was distilled from sodium wire at reduced pressure and stored over molecular sieves. HMPA and HMPT were distilled from $\mathrm{CaH}_{2}$ at reduced pressure and stored over molecular sieves. Hexane solution of nbutyllithium31 and solid phenyllithium32 were prepared as described previously. The concentration of n-butyllithium and phenyllithium solutions were determined by a modification of the double titration method 33 as described below.

Double titration (modification). An aliquot $(1 \mathrm{ml})$ of $\mathrm{PhLi}$ was added by syringe to water (30 $\mathrm{ml}$. aprox.), and the mixture was titrated with $\mathrm{HCl}(0.1 \mathrm{M})$, using phenolphtaleine as indicator. A second aliquot $(1 \mathrm{ml})$ was treated with $0.5 \mathrm{ml}$ of 1,2-dibromoethane, in a $10 \mathrm{ml}$ septum capped round bottoned flask, filled with nitrogen, containing $5 \mathrm{ml}$ of anhydrous hexane, at $50 \mathrm{o}$. The 
mixture was then hydrolized with water and titrated with $\mathrm{HCl} /$ phenolphtaleine. The last value of titration was substracted from the first, obtaining the title of lithiated compound.

Reaction of PhLi with E-cinnamaldehyde. Typical procedure. In a $25 \mathrm{ml}$ septum-capped round-bottoned flask provided with magnetic-stirring and containing $15 \mathrm{ml}$ of THF, under nitrogen, at room temperature, was added the necessary amount of THF solution of PhLi (approx. 1M). Then the additive (when corresponded) and E-cinnamaldehyde (126 $\mu \mathrm{l}, 1 \mathrm{mmol}$.) were added. After $1.5 \mathrm{~h}$ and $3 \mathrm{~h}$ of starting the reaction, a $2 \mathrm{ml}$ aliquot of solution was taken by syringe and injected in a small septum-capped tube, under nitrogen, methanol was added to quench the reaction, the resulting solution was diluted to a final volume of $5 \mathrm{ml}$ with methanol, filtered, and analyzed by $\mathrm{GC}$, using the following conditions: detector temp. $300^{\circ} \mathrm{C}$; injector temp. $250^{\circ} \mathrm{C}$; oven temp $70-280^{\circ} \mathrm{C}\left(12{ }_{\circ} \mathrm{C} / \mathrm{min}\right.$ rate $)$, final time $35 \mathrm{~min}$.

\section{Acknowledgements}

AJV is a grateful recipient of a CONICET (National Research Council from Argentine) fellowship. The authors acknowledge financial support from the University of Buenos Aires and the CONICET.

\section{References}

1. (a) Seebach, D. Angew. Chem., Int. Ed. 1988, 27, 1624. (b) Seebach, D. In Proceedings of the Robert A. Welch Foundation Conferences on Chemistry and Biochemistry; Wiley: New York, 1984.

2. (a) Langer, P.; Freiberg, W. Chem. Rev. 2004, 104, 4125. (b) Mangelinckx, S; Giubellina, N.; De Kimpe, N. Chem. Rev. 2004, 104, 2353. (c) Wang, Q.; Deredas, D.; Huyuh C.; Schlosser, M. Chem. Eur. J. 2003, 9, 570. (d) Snieckus, V. Chem. Rev. 1990, 90, 879. (e) Streitwieser, A.; Wang, D.Z.-R. J. Am. Chem. Soc. 1999, 121, 6213.

3. (a) Thompson, A.; Corley, E. G.; Huntington, M. F.; Grabowski, E. J. J.; Remenar, J. F.; Collum, D. B. J. Am. Chem. Soc. 1998, 120, 2028. (b) Kremer, T.; Harder, S.; Junge, M.; Schleyer, P. v. R. Organometallics 1996, 15, 585

4. McNeil, A. J.; Collum, D. B. J. Am. Chem. Soc. 2005, 127, 5655, and refs. therein.

5. Gregory, K.; Schleyer, P. v. R.; Snaith, R. Adv. Inorg. Chem. 1991, 37, 47, and refs. therein.

6. (a) Nudelman, N. S.; Schulz, H.; Liñares, G. G.; Bonatti, A.; Boche, G. Organometallics 1998, 17, 146. (b) Schulz, H.; Nudelman, N. S.; Viruela-Martin, P.; Viruela-Martin, R.;Tomas Vert, F. J. Chem. Soc., Perkin Trans 2 2000, 1619.

7. Nudelman, N. S.; Liñares, G. G. J. Org. Chem. 2000, 65, 1629, and refs. therein.

8. Rutherford, J. L.; Hoffmann, D.; Collum, D. B. J. Am. Chem. Soc. 2002, 124, 264.

9. Nudelman, N. S.; García, G. V. J. Org. Chem. 2001, 66, 1387 
10. Waldmann, H., "Domino Reactions" In Organic Synthesis Highlight II, p193. H. Waldmann, VCH: Weinheim, 1995.

11. Tietze, L. F. Chem. Rev. 1996, 96, 115.

12. (a) García, G. V; Nudelman, N. S. Int. Org. Prep. \& Proc. 2003, 35, 445. (b) Vázquez, A. J.; Waisbaum, R. G.; Nudelman, N. S. "Organolithium as useful intermediates for Tandem Reactions" Ch. 2 In The Chemistry of Organolithium Compounds, Vol. 2 Rappoport, Z.; Marek, I. Eds.; Wiley: New York, 2005 , in press.

13. Nudelman, N. S.; Shulz, H.G.; García, G. V. J. Phys. Org. Chem. 1998, 11, 722.

14. (a) Reich, H. J.; Green, P. D.; Medina, M. A.; Goldenberg, W. S.; Gudmundsson, B. Ö.; Dykstra, R. R.; Phillips, N. H. J. Am. Chem. Soc. 1998, 120, 7201; (b) Bauer, W.; Winchester, W. R.; Schleyer, P. von R. Organometallics 1987, 6, 2371, and references therein.

15. Reich, H. J.; Goldenberg, W. S.; Sanders, A. W.; Tzschucke, C. C. Org. Lett. 2001, 3, 33.

16. Bauer, W. "NMR of Organolithium Compounds: General aspects and application of twodimensional heteronuclear Overhauser effect Spectroscopy (HOESY)" Ch. 5 In Lithium Chemistry. A theoretical and Experimental Overview Sapse A.-M, Schleyer , P. R. Eds.; Wiley-Interscience: New York, N.Y., 1995.

17. Collum, D. B. Acc. Chem. Res. 1992, 25, 448

18. Qu, B.; Collum, D. B. J. Am. Chem. Soc. 2005, 127, 10820.

19. Davies, R. P.; Raithby, P. R.; Snaith, R. Angew. Chem., Int. Ed. 1997, 36, 1215.

20. Armstrong, D. R.; Davidson, M. G.; Davies, R. P.; Mitchell, H. J.; Oakley, R. M.; Raithby, P. R.; Snaith, R.; Warren, S. . Angew. Chem., Int. Ed. 1996, 35, 1942.

21. Williard, P. G.; Liu, Q. Y.; Lochmann, L. J. Am. Chem. Soc. 1992, 114, 348.

22. Yamataka, H.; Kawafuji, Y.; Nagareda, K.; Miyano, N.; Hanafusa, T. J. Org.Chem. 1989, 54, 4706.

23. Ashby, E.; Coleman, D.; Gamasa, M. J. Org. Chem. 1987, 52, 4079.

24. Nudelman, N. S.;Doctorovich, F. Tetrahedron 1994, 50, 4651.

25. Nudelman, N. S.; Schulz, H. G. J. Chem. Soc., Perkin Trans. 2, 1999, 2761

26. (a) Reich, H. J.; Sikorski, W. H. J. Org. Chem. 1999, 64, 14. (b) Sikorski, W. H.; Reich, H. J. J. Am. Chem. Soc. 2001, 123, 6527.

27. Carlier, P. R.; Lo, C. W.; Lo, M. M.; Wan, N. C.; Williams, I. D. Organic Letters 2000, 2, 2443

28. Reich, H. J.; Sanders, A. W.; Fiedler, A. T.; Bevan, M. J. J. Am. Chem. Soc. 2002, 124, 13386

29. Romesberg, F. E.; Collum, D. B. J. Am. Chem. Soc. 1995, 117, 2166.

30. Shriver, D. F. The Manipulation of Air-Sensitive Compounds, Wiley: New York, 1989.

31. Nudelman, N. S.; Vitale, A. A. Org. Prep. Proc. Int. 1981, 13, 144.

32. Nudelman, N. S.; García, G. G.; Velurtas, S. J. Phys. Org. Chem. 2002, 15, 903.

33. Gilman, H.; Cartledge, F. K. J. Organomet. Chem. 1964, 2, 447. 